\title{
Gesundheit um jeden Preis - aber wer bezahlt?
}

\author{
Jacques de Haller \\ Dr. med., Präsident des Komitees forumsante.ch
}

\begin{abstract}
Die Finanzierung unseres Gesundheitswesens befindet sich in einer Sackgasse. Weil die bisherigen Quellen an ihre Grenzen stossen, muss die Schweiz gezwungenermassen neue Formen der Finanzierung der Gesundheitsversorgung der Bevölkerung finden. Welche Modelle kommen hierzu in Frage? Und welche Auswirkungen haben sie auf die verschiedenen Akteure und Nutzer?
\end{abstract}

Wachsende Bedürfnisse und eine der höchsten Direktfinanzierungsraten (out-of-pocket) Europas (vgl. OECD: http://dx.doi.org/10.1787/health-data-en) führen das schweizerische Gesundheitswesen in eine Sackgasse: Die Versicherten sind mehrheitlich nicht imstande, zunehmend höhere Beiträge zu leisten, und auch die Mittel der öffentlichen Hand sind limitiert. Es soll nicht um ideologische oder polemische Äusserungen gehen, sondern darum, ein Problem zu beleuchten, dessen Brisanz immer deutlicher zu Tage tritt und das es zu diskutieren gilt, solange es noch überschaubar ist. Natürlich könnte man die Kosten beklagen, die hierzulande wie in der gesamten westlichen Welt von Jahr zu Jahr ansteigen. Dies ändert jedoch nichts an der Tatsache, dass es nicht möglich ist, die Kosten zu senken oder gar nur zu stabilisieren, ohne unser Gesundheitswesen komplett umzubauen oder zumindest einige seiner grundlegenden Prinzipien aufzugeben. Dies wäre nicht nur falsch, die Bevölkerung würde es auch nicht akzeptieren.

Eine Reihe von Anzeichen weisen auf den prekären $\mathrm{Zu}$ stand des aktuellen Finanzierungssystems hin. Allein die Tatsache, dass immer mehr Prämienverbilligungen ausbezahlt werden müssen, deutet darauf hin, dass das aktuelle System zunehmend ad absurdum geführt wird. Mittlerweile sind, je nach Kanton, bis zur Hälfte der Versicherten (NW) auf eine Prämienverbilligung von bis zu 6000 Franken pro Jahr pro Haushalt (AR) angewiesen (schweizerischer Durchschnitt für das Jahr 2014: 3118 Franken pro Jahr; 27\% der Bevölkerung) (BSV, www. bsv.admin.ch/dokumentation/).
Dr. med. Jacques de Haller

12, chemin de Seppey

CH-1085 Vulliens

mail[at]jdehaller.ch
Das zögerliche, wenig kohärente Vorgehen unserer Gesundheitspolitik ist ein Spiegel der vertrackten Situation, in der wir uns befinden. Zwischen «reguliertem Wettbewerb» und freier Marktwirtschaft hin- und herpendelnd, wird nach Wegen gesucht, die Gesundheitskosten einzudämmen, in Ermangelung von Lösungen die langfristige Finanzierung zu sichern. Diese Unentschlossenheit erklärt zweifellos zu einem grossen Teil die Unfähigkeit, wirksame Entscheide zu treffen.

Einfache Lösungen gibt es nicht: Die langfristige Finanzierung eines Gesundheitswesens zu sichern, dessen Ausgaben jährlich um mehrere Prozentpunkte ansteigen, bedeutet, dass man entweder zusätzliche Geldquellen erschliessen oder die Leistungen einschränken muss. Verständlich, dass unsere Politiker/-innen vor solchen Entscheiden zurückschrecken. Die Option, das Gesundheitsbudget vermehrt über Steuern zu finanzieren, indem die Prämienbelastung durch zusätzliche - direkte oder, wie im Fall anderer Sozialversicherungen, indirekte - Steuern abgefedert wird, ist für die Mehrheit des gegenwärtigen Parlaments ein Schreckgespenst. Den freien Zugang zur Gesundheitspflege einzuschränken, respektive den Katalog der OKP-Leistungen zu kürzen, ist für das linke Lager ein «No-go», da sich der Zugang zu Gesundheitsleistungen vermehrt nach der wirtschaftlichen Leistungsfähigkeit der Individuen richten würde, was als ungerecht empfunden wird. Es ist somit wichtig, eine offene Diskussion zu diesem Thema zu lancieren, um innovative, gerechte und nachhaltige Lösungen zu finden.

Die Gewissheit, im Bedarfsfall die benötigten medizinischen und pflegerischen Leistungen zu erhalten, unabhängig von der wirtschaftlichen und sozialen Lage, in der man sich zu diesem Zeitpunkt befinden mag, ist eine grundlegende Errungenschaft des 20. Jahrhunderts und zugleich ein Garant der Stabilität der westlichen Gesellschaft. Das dürfen wir nicht vergessen. Diese Errungenschaft hat einen Preis - doch wer wird ihn in Zukunft bezahlen? 